\section{$\underset{\substack{\text { hommes } \\ \text { \& migrations }}}{ }$}

\section{Hommes \& migrations}

Revue française de référence sur les dynamiques

migratoires

\section{$1283 \mid 2010$}

Cuisines et dépendances

\title{
Les migrations polonaises en Grande-Bretagne et en Irlande
}

après l'élargissement à l'Est de l'UE et leur impact en Pologne

Anne de Tinguy et Catherine Wihtol de Wenden

\section{(2) OpenEdition}

Journals

Édition électronique

URL : http://journals.openedition.org/hommesmigrations/1014

DOI : 10.4000/hommesmigrations. 1014

ISSN : 2262-3353

Éditeur

Musée national de l'histoire de l'immigration

Édition imprimée

Date de publication : 1 janvier 2010

Pagination : 169-179

ISBN : 978-2-919040-04-9

ISSN : 1142-852X

Référence électronique

Anne de Tinguy et Catherine Wihtol de Wenden, « Les migrations polonaises en Grande-Bretagne et en Irlande », Hommes \& migrations [En ligne], 1283 | 2010, mis en ligne le 01 janvier 2013, consulté le 15 septembre 2020. URL : http://journals.openedition.org/hommesmigrations/1014

Ce document a été généré automatiquement le 15 septembre 2020.

Tous droits réservés 


\title{
Les migrations polonaises en Grande-Bretagne et en Irlande
}

\author{
après l'élargissement à l'Est de l'UE et leur impact en Pologne
}

Anne de Tinguy et Catherine Wihtol de Wenden

1 Officiellement, entre 2002 et fin 2007, quelque 2,3 millions de Polonais sont partis à l'étranger, dont 1,9 million dans un pays de l'UE. Les flux sont probablement plus importants, un certain nombre de personnes s'y étant installées avant le $1^{\mathrm{er}}$ mai 2004 sans permis de travail. Ces flux s'inscrivent dans un contexte d'accroissement des migrations vers l'Europe des Quinze, quand les instances européennes et les pays d'accueil ont progressivement pris conscience, début 2000, de leur déclin démographique et des pénuries sectorielles de main-d'œuvre (agriculture, services, restauration, hôtellerie, bâtiment, informatique, médecine).

2 L'élargissement de l'UE à l'Est en 2004 produit une nouvelle vague de départs. La Grande-Bretagne et l'Irlande attirent des gens jeunes, éduqués, qui continuent traditionnellement à venir de milieux ruraux et de petites villes, mais qui sont plus nombreux à venir de grandes villes. Ces nouveaux arrivants sont très vite perçus positivement dans ce que les Polonais appellent "les îles". La peur du "plombier polonais", qui a été si vive en France, ne s'est guère répandue en Grande-Bretagne ni en Irlande.

3 En Pologne, l'émigration est difficile à évaluer car, depuis 2004, la plupart des migrants polonais ne déclarent pas leurs sorties (537 000 se trouvaient à l'étranger depuis plus de deux mois au second trimestre 2007, dont une forte proportion de femmes). Durant les dix dernières années, $9 \%$ des adultes polonais ont travaillé à l'étranger, soit 2800000 personnes et $1 \%$ déclare y travailler actuellement. $52 \%$ des personnes qui déclarent avoir travaillé à l'étranger sont parties de Pologne après le $1^{\text {er }}$ mai 2004 et $19 \%$ disent avoir quitté la Pologne avant 2004 et continuer à travailler à l'étranger. La Suède, l'Allemagne et la Norvège attirent aussi de nombreux candidats. En Grande-Bretagne, les Polonais sont les plus nombreux parmi les étrangers : 406000 en 2007, dont 292000 pourvus d'un emploi. En Irlande, leur nombre est estimé à 350000 et les Polonais formaient, en 2006, avec les autres ressortissants des PECO, $42 \%$ des entrants ${ }^{1}$. En 2007, 
une "carte verte" pour les métiers très qualifiés et les secteurs moins qualifiés connaissant une pénurie de main-d'œuvre, ainsi qu'un dispositif de prolongation du séjour pour les diplômés de l'enseignement supérieur, a continué à rendre l'Irlande très attractive.

\section{De l'élargissement de l'Union européenne à l'ouverture économique}

Bien que la liberté de circulation soit l'un des droits fondamentaux des Européens, les processus d'élargissement européens se sont souvent accompagnés de périodes d'attente entre l'entrée de nouveaux membres et l'accès à la pleine liberté de circulation, d'installation et de travail des ressortissants des nouveaux États.

Pour les pays d'Europe centrale et orientale (PECO), un processus graduel a été mis en place, qui tient compte du choix par chaque pays de l'Europe des Quinze de la date et des pays auxquels il accorde la liberté de travail et d'installation, en fonction de la situation de son marché du travail, en trois phases. Les pays de l'Europe des Quinze peuvent accorder ces droits dès l'entrée des nouveaux États, comme ce fut le cas du Royaume-Uni, de l'Irlande et de la Suède à l'égard des Polonais. Ils peuvent attendre une période supplémentaire de deux ans, motivée, puis une seconde période de trois ans, encore motivée, et, enfin, encore deux ans s'ils ne sont pas prêts à affronter la concurrence. La durée maximum d'attente est ainsi de sept ans. La France a choisi la fin de la période de sa présidence de l'Union européenne, en 2008, pour accorder la liberté de travail et d'installation aux ressortissants des PECO, sauf à la Bulgarie et à la Roumanie.

6 L'ouverture précoce par la Grande-Bretagne, l'Irlande ainsi que la Suède, le $1^{\mathrm{er}}$ mai 2004, de leur marché du travail aux PECO a eu pour effet un afflux de migrants polonais dans les deux premiers pays. Après la chute du mur de Berlin, les Polonais étaient venus nombreux en Allemagne, pendant les trois mois de libre circulation qui leur étaient alloués (commerçants "à la valise" et migrants temporaires). Ils sont aussi allés travailler au noir en Italie, à la faveur des réseaux catholiques polonais consolidés à Rome par Jean-Paul II, comme gardes d'enfants et de personnes âgées (badanti).

Entre 2002 et 2007, 690000 Polonais se sont installés en Grande-Bretagne et 200000 en Irlande. Des ressortissants d'autres PECO s'installent également en Grande-Bretagne, mais les Polonais sont de loin les plus nombreux, devant les Indiens.

En 2006, les statistiques polonaises estimaient que $30 \%$ des migrants polonais vivaient dans ce pays, la seconde destination étant l'Allemagne (23\%), qui a officiellement accueilli 490000 Polonais durant la même période.

Il s'agit d'une population jeune ( $82 \%$ des personnes ayant entre 18 et 34 ans), majoritairement célibataire, dont le niveau d'éducation est élevé.

La moyenne d'âge des plus de 15 ans partis à l'étranger pour une durée de plus de deux mois y est de 25 ans, contre 46 ans aux États-Unis. Un migrant polonais sur cinq a un diplôme universitaire. Les Polonais migrent plus que les Polonaises : 57 \% d'hommes pour $43 \%$ de femmes et $56 \%$ de personnes mariées ou vivant en couple ${ }^{2}$. Les départs ont, pour $31 \%$, concerné les jeunes actifs habitant les grandes villes de plus de 200000 habitants. La plupart soutiennent leur famille ou économisent pour investir en Pologne, notamment dans l'achat d'un logement. Les raisons du départ sont le travail (51 \%) et la 
recherche d'un travail (42\%), la volonté de gagner davantage (42\%), l'apprentissage d'une langue $(20 \%)$, le désir de vivre une expérience $(17 \%)$ et la volonté d'aider la famille en Pologne (14\%).

11 Ces Polonais ont trouvé du travail dans le bâtiment, les services (entretien, restauration), l'agriculture, où la demande de main-d'œuvre est forte et l'offre insuffisante. Si les emplois sont souvent peu payés, dans l'agriculture, les salaires anglais représentent le triple de ceux de Pologne et dans le bâtiment, le double ${ }^{3}$. Bien que leur part dans le marché du travail ait eu un impact faible ou nul sur les salaires des autochtones, la controverse sur la concurrence déloyale des Polonais a été relancée par la crise. En février 2009, des grèves ont éclaté contre l'emploi de travailleurs étrangers sur plus d'une douzaine de sites énergétiques avec pour slogan UK jobs for British workers ("Des emplois anglais pour des travailleurs anglais").

\section{Les raisons du départ}

12 Les raisons économiques ne sont pas les seules à être à l'origine de cette migration, mais elles jouent un rôle essentiel. Le taux de chômage des régions de départ était, en juin 2005, de 27,6 \% en Warmie-Mazurie, 25,5\% en Poméranie occidentale, 22,5\% en Kujawy-Poméranie, 24,4 \% en Lubuskie, 21,1 \% en Basse-Silésie. En 2007, les taux étaient encore en Warmie-Mazurie de $18,7 \%$ et en Poméranie occidentale de $16,3 \%$, régions où le chômage est le plus élevé, malgré une croissance prévue de 4,9\% entre 2007 et 2015. 12 millions de Polonais vivent à la limite de la pauvreté, avec moins de 2,5 euros par jour. Le désenchantement et le manque de confiance dans les gouvernants entrent aussi en ligne de compte. Les facilités de transport entre les îles Britanniques et la Pologne ont également accompagné le mouvement.

Une autre raison est liée à la capacité des Polonais à être très mobiles pour répondre aux besoins de main-d'œuvre. Certains se sont insérés dans les zones rurales où le recrutement est difficile. Durant ces quatre dernières années, $39 \%$ travaillaient dans l'administration, les affaires et la gestion, 19 \% dans les hôpitaux et le nettoyage, 10 \% dans l'agriculture, $7 \%$ dans les usines, $5 \%$ dans les circuits de la viande et du poisson, salariés à $86 \%$ et à leur compte à $14 \%$. D'autres travaillent comme saisonniers dans l'agriculture les mois d'été (sud-est du pays).

14 Ces migrants subissent une forte déqualification entre les niveaux d'éducation acquis et les emplois occupés. 70\% n'utilisent pas leurs qualifications. Ils aspirent à gagner davantage que chez eux en peu de temps, même dans des métiers peu qualifiés. Habitués à des emplois qualifiés ou peu qualifiés, ceux qui sont venus pour chercher un travail et améliorer leur niveau de vie finissent dans des emplois très peu prisés, par manque de connaissance du marché du travail local et de la langue ${ }^{4}$. Parmi les métiers typiques exercés par les Polonais, on retrouve ceux de boucher, conditionneur de poisson, ingénieur, jardinier, travailleur du bâtiment, employé dans le tourisme (Écosse). 22 \% se considèrent comme saisonniers. Un tiers avaient l'intention de rester moins de deux ans, mais un quart ne savent pas combien de temps ils vont rester.

Parce qu'elle touche un pourcentage significatif de la population, notamment les jeunes actifs, qu'elle concerne fortement certaines régions et qu'elle est suivie de retours de différentes sortes, la vague migratoire de l'après-élargissement ne peut que marquer la société polonaise et avoir de multiples répercussions. Elle est une voie dans 
l'intégration des Polonais au sein de l'UE, modifiant les perceptions mutuelles et les rapports que les Polonais entretiennent avec les autres Européens.

Ce phénomène a une forte prégnance et une grande visibilité en Pologne. À la fin de 2006, il touchait déjà $12 \%$ de la population active. Dans 7,3\% des 14 millions de foyers polonais, au moins un membre de la famille travaille à l'étranger et bien davantage dans certaines voïvodies : $14,7 \%$ dans celle de Subcarpathie et $13,1 \%$ dans celle d'Opole. Dans cette dernière région, un habitant sur cinq a travaillé à l'étranger au cours des dix dernières années. Certaines régions sont, en outre, particulièrement touchées par la migration des jeunes : la voïvodie de Warmie-Mazurie a perdu $24,4 \%$ de ses jeunes, celle de Sub-Carpathie, $21,1 \%$, celle de Podlasie, $21,5 \%$ et celle de Lublin, $18,1 \%{ }^{5}$.

La migration fait ainsi partie de la vie quotidienne de nombreux Polonais. Très présente dans les médias, elle a, en outre, fait irruption dans le débat politique national. Des émissions de télévision lui sont consacrées, comme la série Londynczycy (Les Londoniens), depuis octobre 2008. L'Angleterre est volontiers désignée comme la $17^{\mathrm{e}}$ voïvodie de Pologne. Le terme de "deuxième Irlande", synonyme de réussite de l'intégration dans l'espace européen, est entré dans le langage politique.

\section{Intégration et jeu des représentations dans l'espace européen}

La géographie des flux migratoires polonais après l'élargissement est révélatrice de la place que l'UE prend dans l'évolution de la Pologne. Alors que l'Union accueillait $52 \%$ des migrants polonais en 2002, elle en accueille près de $80 \%$ entre 2004 et 2006 : la part des pays européens augmente fortement au détriment d'autres destinations, en particulier de l'Amérique du Nord. Au sein de l'UE, à la relation forte qui existe depuis vingt ans entre la Pologne et l'Allemagne s'ajoute désormais un nouveau "couple" privilégié avec la Grande-Bretagne et l'Irlande. Cette réorientation des flux est un coup d'arrêt donné à une grande tradition d'émigration vers les États-Unis ${ }^{6}$.

$\mathrm{Au}$ moment de leur adhésion à l'Union, par la migration, les Polonais renforcent et développent la place qu'ils occupent dans l'espace européen, car leur intégration dans le marché du travail s'accompagne de celle de leur famille. Fin 2007, sur les 240000 enfants de familles est-européennes ou centre-européennes qui sont scolarisés en Grande-Bretagne, 170000 sont Polonais. Et le nombre croissant de naissances d'enfants de parents polonais sur le sol britannique alimente ce phénomène (1 393 en 2003, 6620 en 2006) ${ }^{7}$. Certains commencent à s'impliquer dans la vie politique de leur pays d'accueil. En Irlande, des Polonais sont candidats aux élections municipales de juin $2009^{8}$. Au Royaume-Uni, ils ont formé des associations d'aide aux compatriotes.

L'image des Polonais en Grande-Bretagne et en Irlande s'est elle aussi améliorée, même si l'arrivée des Polonais a parfois créé des tensions quand ils se sont installés en nombre dans de petites agglomérations. Il n'en reste pas moins que cette vague d'émigration a très positivement influé sur l'image des Polonais et de la Pologne, mal connue en 2004. En Allemagne, en septembre 2008, l'image des Polonais est associée au pouvoir de l'Église, à la corruption et à la bureaucratie ${ }^{9}$. Les travailleurs polonais en GrandeBretagne ouvrent la voie à de nouvelles perceptions: travailleurs, consciencieux, disciplinés, responsables et donc fiables, ayant à la fois le sens du travail et celui de la famille à laquelle ils sont très attachés, ils sont en général très appréciés ${ }^{10}$. 
21 Les employeurs britanniques sont nombreux à préférer confier certains postes, peu rémunérés et qualifiés, à des Européens de l'Est plutôt qu'à des Britanniques, les premiers étant considérés comme moins exigeants, plus motivés, plus fiables, plus ponctuels, moins absentéistes que les Britanniques.

La migration contribue aussi à modifier les représentations de l'Europe en Pologne. Tadeusz Mazowiecki, ancien chef du gouvernement, note, dès janvier 2006, que "l'image de l'Europe en Pologne a évolué de façon très positive... : aujourd'hui, on ne se sent plus menacé par les catastrophes que notre adhésion aurait soi-disant provoquées ${ }^{11}$ ". Le rapport de la Pologne à l'UE reste complexe. Et pourtant, comme le résume en 2008 un observateur : "En dépit de son étiquette eurosceptique, la Pologne est un pays peuplé d'euro-enthousiastes. Le taux des partisans de l'intégration européenne a atteint en juillet 2007 le niveau record de $89 \%$ $(\mathrm{CBOS})^{12}$."

23 L'immigration, à laquelle la Pologne doit désormais avoir recours, permet de son côté de densifier l'espace européen, en recréant ou confortant les liens avec l'Ukraine, la Biélorussie et la Russie. Aux tensions lors de l'entrée de la Pologne dans l'UE avec ses voisins est-européens succèdent de nouvelles solidarités. Les départs provoquant des pénuries de main-d'œuvre, le gouvernement polonais fait appel à des travailleurs étrangers, qui viennent principalement des pays de l'ex-URSS et d'Asie (Chine et Vietnam).

Une loi entrée en vigueur le 29 mars 2008 instaure "une carte de Polonais", destinée à des ressortissants des États de l'ex-URSS dont au moins un des parents ou grandsparents est ethniquement Polonais. Valable dix ans, celle-ci donne accès au marché du travail polonais ainsi qu'au système éducatif. Une directive du ministère du Travail du 20 juillet 2007 autorise les ressortissants des pays voisins (Ukrainiens, Russes et Biélorusses) à travailler six mois sans permis de travail ${ }^{13}$. Varsovie signe le 28 mars 2008 avec l'Ukraine un accord sur les mouvements frontaliers : un permis spécial, valable deux ans, permet aux Ukrainiens des régions frontalières de passer la frontière et de rester sur le territoire polonais jusqu'à soixante jours. D'autre part, en 2008, la délivrance des permis de travail à des étrangers est simplifiée et les permis sont accordés pour trois ans (au lieu de deux).

\section{Les conséquences économiques de la migration}

La vague migratoire de l'après-2004 a participé à la relance de l'économie polonaise. Au cours des deux années qui ont précédé l'entrée de la Pologne dans l'UE, le chômage touchait environ $20 \%$ de la population. À partir de 2004, il décroît continûment pour tomber, en 2008 , à $9 \%$. Le lien avec la vague d'émigration ne fait guère de doute : la baisse du chômage est la conséquence de l'ampleur des départs ${ }^{14}$. Parallèlement, les salaires augmentent - de $2 \%$ entre 2004 et 2006, la progression est de $9 \%$ en 2007 -, les migrations provoquant une forte diminution de l'offre de main-d'œuvre dans certains secteurs ${ }^{15}$.

La Pologne est encore loin d'avoir "rattrapé" le niveau de vie du reste de l'UE : en 2007, le PIB par habitant est de 7966 dollars en Pologne, de 33915 en France et de 30112 en Italii $^{16}$. Mais le taux de satisfaction de la population augmente. Lors d'une enquête faite par CBOS en septembre-octobre $2007^{17}$, une personne interrogée sur quatre se dit 
satisfaite de ses conditions de vie matérielles et près des trois quarts des personnes s'estiment satisfaites de leur existence.

Les transferts financiers faits par les Polonais qui travaillent à l'étranger contribuent àcette embellie. En 2006, 17,7 milliards de zlotys ont été transférés en Pologne ; en 2007, 20 milliards (soit deux fois plus qu'en 2004). L'Irlande (5 milliards de zlotys) et la Grande-Bretagne (4,6 milliards) viennent en tête des pays d'origine des fonds transférés, les États-Unis et l'Allemagne ne venant que loin derrière. Pour faciliter les mouvements de capitaux, la Banque de Pologne a signé en juillet 2007 un accord de coopération avec une des grandes banques britanniques - la Nat West. Toutefois, il faut noter que ces transferts favorisent davantage la consommation que les transformations structurelles de l'économie. En Grande-Bretagne et en Irlande, l'argent envoyé en Pologne est principalement destiné à la famille : consommation courante et logement. La part des investissements, matériels ou immatériels, ne vient qu'ensuite ${ }^{18}$.

Des transferts de compétence vers la Pologne ont bien lieu. Travailler en GrandeBretagne ou en Irlande, où le travail individuel est à la base de la réussite professionnelle, a rendu les Polonais plus responsables, moins demandeurs et davantage désireux de formation. D'où l'idée exprimée par certains en Pologne que celle-ci a tout à gagner à tirer profit de l'expérience acquise par les émigrés à l'étranger et à les associer à la modernisation du pays ${ }^{19}$. Mais ce point de vue ne correspond pas toujours à la réalité, loin s'en faut. Des Polonais qui ont créé une entreprise en GrandeBretagne ou en Irlande ne semblent guère prêts à transposer leur expérience en Pologne en raison des complexités administratives dans ce pays. La presse s'est faite l'écho des "pièges juridiques" qui attendent ceux qui reviennent ${ }^{20}$. Le schéma le plus fréquent est celui d'une difficile réintégration du marché du travail polonais, pour des raisons liées à la nature de l'expérience en Grande-Bretagne, aux objectifs que les émigrés poursuivaient en s'y installant, aux postes qu'ils ont occupés, à la déqualification qu'ils ont été amenés à accepter et à leur mode de vie à l'étranger.

Les départs ont par ailleurs provoqué sur le marché du travail des tensions qui brident l'économie polonaise. Ils sont, dès 2005, à l'origine de pénuries de main-d'œuvre qui s'aggravent en 2007. C'est le cas dans le bâtiment, l'industrie manufacturière (agroalimentaire, travail des métaux, habillement) et dans le secteur médical (anesthésie, radiologie, chirurgie plastique) ${ }^{21}$. Si l'on en croit un article de Dziennik, au début de 2009, $20 \%$ des médecins polonais seraient partis travailler à l'étranger, en Angleterre, en Allemagne et, dans une moindre mesure, en Irlande ${ }^{22}$. Dans le bâtiment, les tensions se font d'autant plus sentir que les émigrés sont nombreux à vouloir y investir. Elles peuvent en outre compliquer certains grands projets, comme celui de l'Euro 2012: le manque d'électriciens, de mécaniciens et d'ouvriers qualifiés se répercute sur la préparation de cette grande manifestation sportive qui implique de construire autoroutes, stades et hôtels ${ }^{23}$. Elles contribuent à la hausse des salaires déjà évoquée, ce qui risque d'affaiblir l'attractivité de la Pologne aux yeux des investisseurs étrangers ${ }^{24}$.

30 Enfin, sur le plan démographique, la vague d'émigration contribue à la crise de la natalité en Pologne (720 000 naissances en 1984, 374000 en 2006), à un moment où le taux de fécondité s'établit à 1,24 enfant par femme (donnée de 2005), l'un des taux les plus bas de l'UE et, à terme, à une diminution significative de la population. Entre l'adhésion de 2004 et le $1^{\text {er }}$ janvier 2007, la Pologne enregistre une perte nette de population de près de 1,1 million de personnes (soit $2,8 \%$ de la population totale), dont 
1,01 million sont âgées de 15 à 59 ans, ce qui représente $4 \%$ de la population de cette tranche d'âge. Si les hommes jeunes partis à l'étranger ne reviennent pas, "la question du renouvellement de la population se posera plus vite que prévu" et le vieillissement de la population s'accentuera ${ }^{25}$. Si les autorités ne parviennent pas à attirer une immigration soutenue, ces tensions démographiques auront inévitablement pour effet d'accentuer celles qui se font déjà sentir sur le marché du travail ${ }^{26}$.

\section{La mise en place d'une politique de retour?} amélioration de leurs qualifications professionnelles ou de leurs perspectives de carrière. Ils ne peuvent pas prétendre aux postes de haut niveau que leurs études en Pologne auraient pu leur ouvrir. L'adaptation de leurs qualifications aux besoins $d u$ marché polonais est un autre facteur d'incertitude ${ }^{27}$. Certains, jeunes, diplômés et maîtrisant bien l'anglais, se retrouvent ainsi doublement exclus. En Grande-Bretagne, ils étaient frustrés par un travail en-dessous de leurs qualifications. En Pologne, ils peinent à se réinsérer dans le marché du travail. Ils se sentent trop Anglais pour la Pologne, trop Polonais pour l'Angleterre, et les années passées à l'étranger ne sont pas à l'origine d'un nouveau bagage de compétences qui permettrait au retour une carrière plus rapide et plus intéressante ${ }^{28}$. novembre 2008, Donald Tusk, premier ministre, a lancé un programme intitulé : “As-tu un plan pour le retour ?" Au même moment est publié un ouvrage-conseil de 177 pages appelé "Powrotnik - Guide pour ceux qui reviennent", disponible dans les consulats, les associations, dans la presse et sur le site du même nom (www.prowroty.gov.pl) : il donne des réponses pratiques aux questions que se posent ceux qui veulent revenir ou qui s'interrogent sur l'opportunité d'un retour (retraites, assurances, écoles, etc.) ${ }^{29}$. D'autres initiatives ont été prises, soit au niveau national, soit au niveau local. Un projet "12 villes - Revenir, mais où ?" a été lancé en janvier 2009 par l'association Poland Street avec douze agglomérations: Bialystok, Bydgoszcz, Gdansk, Katowice, Cracovie, Lublin, Lodz, Poznan, Rzeszow, Szczecin, Varsovie et Wroclaw. Il vise à mettre en contact les émigrés avec les pouvoirs locaux et des responsables économiques. Des séminaires, auxquels participent des représentants polonais de chambres de commerce, des entrepreneurs locaux, etc. sont organisés à Londres par chacune de ces municipalités pour tenter d'encourager les émigrés à revenir en Pologne. Le ministère polonais de la Défense, qui cherche à recruter, a fait part de son désir de se joindre à cette action $^{30}$.

Les observateurs sont nombreux à souligner les limites de l'action polonaise à l'égard des Polonais de l'étranger. La Banque mondiale a critiqué, dans un de ses rapports sur le thème, l'absence d'une politique cohérente concernant les transferts, l'accueil des migrants et celui des Polonais qui reviennent ${ }^{31}$. Plusieurs études demandent la définition d'une politique d'immigration et la presse se fait l'écho, pour le regretter, du peu d'intérêt des autorités polonaises pour le formidable potentiel que représentent les Polonais de l'étranger ${ }^{32}$. 


\section{La crise financière de 2008-2009 : la fin d'un phénomène}

34 financière mondiale. En Grande-Bretagne, la détérioration de la situation économique et l'augmentation du chômage se sont répercutées sur les migrants polonais, certains se retrouvant dans des situations très difficiles ${ }^{33}$. Ce sont ceux qui effectuent les travaux temporaires et les moins qualifiés qui sont les plus touchés par les licenciements, notamment dans le secteur de la construction. Si l'on en croit la presse britannique ${ }^{34}$, avec la crise, près de 500000 Polonais pourraient revenir au pays. D'après un rapport d'avril 2008 de l'Institute for Public Policy Research de Londres, la moitié des travailleurs polonais arrivés en Grande-Bretagne depuis 2004 auraient quitté le territoire britannique ${ }^{35}$. En Irlande, le nombre des Polonais aurait diminué d'une bonne moitié au cours du deuxième semestre 2008( ${ }^{36}$ ), un phénomène qui ne fait que s'amplifier par la suite. Dans un premier temps, la détérioration du taux de change de la livre sterling rend les transferts financiers en Pologne beaucoup moins lucratifs (la livre, qui s'échangeait contre 7 zlotys en 2004, n'en vaut plus que 4 en 2008, ce qui grève les économies envoyées par les travailleurs polonais dans leur pays ${ }^{37}$ ). L'incitation à partir pour des raisons de différentiel de revenus diminue et rend plus attractif le marché du travail polonais (augmentation des salaires, vacances de postes dans plusieurs secteurs). Dans un deuxième temps, la récession qui s'installe pousse les Polonais à quitter "les îles". La nostalgie de la Pologne, souvent évoquée, achève de convaincre certains de retourner au pays ${ }^{38}$.

Il n'y a pas pour autant d'exode de Polonais fuyant la Grande-Bretagne et l'Irlande. En Pologne, pour la première fois en cinq ans, le taux de chômage a de nouveau augmenté, passant en novembre 2008 à 9,1\%, soit 1,39 million de personnes (il était de $8,8 \%$ en octobre $^{39}$ ). Interrogées en avril 2009, ni les compagnies aériennes (Ryanair, LOT) ni les sociétés d'autocars (Orbis, Eurolines) n'observent de mouvement de masse. Les données sur la scolarisation des enfants polonais à Londres vont dans le même sens: leur nombre dans la capitale britannique a augmenté de $43 \%$ entre mai 2007 et décembre 2008 , et un phénomène du même type a été observé dans d'autres villes ${ }^{40}$. La Fédération des Polonais en Grande-Bretagne, en janvier 2009, estime qu'au moins 500000 Polonais ont l'intention de rester en dans ce pays ${ }^{41}$. En Irlande, la tendance semble être la même : le fait que Polska Gazeta, journal polonais de Dublin, n'ait pas réduit son tirage (10 000 exemplaires) ou que la fréquentation de certains commerces polonais n'ait pas diminué en témoigne ${ }^{42}$.

Beaucoup des Polonais interrogés estiment qu'en cette période il leur est moins difficile de trouver ou retrouver du travail en Grande-Bretagne qu'en Pologne : dans certains cas, ils occupent des emplois dont les Britanniques ne veulent pas; dans d'autres, ils sont préférés par les employeurs à des nationaux, jugés moins motivés, moins fiables et dont les salaires sont plus élevés ${ }^{43}$. Autrement dit, certains employeurs qui doivent licencier préfèrent écarter leurs concitoyens, ceux-ci ayant des salaires plus élevés et une moindre productivité que les Polonais ${ }^{44}$. La baisse des prix dans l'immobilier incite certains à rester, voire, s'ils en ont les moyens, à investir en Grande-Bretagne. 


\section{Le dilemme des autorités polonaises}

37 La vague migratoire en Grande-Bretagne et en Irlande, jusqu'en 2008, a conduit les autorités polonaises à prendre position en faveur du retour de ceux qui étaient partis : c'était le sens du programme "Retour". Le fait que cette vague d'émigration, en raison de son caractère massif et parce qu'elle a concerné de nombreux jeunes diplômés, soit largement perçue comme une perte était une raison supplémentaire pour agir en ce sens.

La dégradation de la situation économique en Pologne conduit nombre de responsables politiques, au niveau national, mais aussi local ou régional, à modifier leurs positions sur la question. Ainsi, en juillet 2008, a été votée une loi supprimant la double imposition des revenus des Polonais travaillant en Grande-Bretagne et en Irlande. Par ailleurs, certains responsables déconseillent désormais aux émigrés de revenir, car "ici, il n'y a pas de travail". Un des responsables de la région d'Opole, Jozef Sebesta, a déclaré à la presse que "cela n'avait aucun sens dans la situation actuelle de tenter de convaincre les gens de revenir", ceux qui revenaient en Pologne se retrouvant immédiatement au chômage. Il en a conclu que le programme mis en place en 2007 Opolskie - tu zostaje (Opolskie - ici je reste) n'avait plus de raison d'être ${ }^{45}$.

D'autres poursuivent leur action en faveur des retours, mais de façon sélective. La ville de Szczecin en janvier 2009 et celle de Poznan en février ont mené une action pour favoriser le retour d'informaticiens, de médecins, de chimistes et de pharmaciens. La ville de Bialystok prévoit des initiatives pour ceux qui seraient prêts à investir dans le tourisme de la région. Gdansk a renoncé à participer à ce projet ${ }^{46}$.

40 L'immigration polonaise en Grande-Bretagne et en Irlande peut être comparée à celle des Portugais en France, qui ont longtemps formé un "couple migratoire" avec la France. Cette migration a permis au Portugal, avant et après son entrée dans l'UE, de moderniser les esprits et de s'ouvrir à l'Europe, sans pour autant entraîner une promotion professionnelle des Portugais concernés. Mais l'expérience migratoire leur a permis de mieux vivre, d'accéder à la consommation, d'ouvrir et de transformer les mentalités.

\section{NOTES}

1. OCDE, SOPEMI 2008.

2. IPPR, 2008.

3. Jacek Tomkiewicz, "Migration and Income Inequalities Both within and between Old and New Members", colloque international sur les migrations, Varsovie, 8 décembre 2008.

4. Stephen Nickell, Jumana Saleheen, "The Impact of Immigration on Occupational Wages: Evidence from Britain", séminaire international sur les migrations, Varsovie, 8 déc. 2008.

5. Beata Roguska, "Le travail des Polonais à l'étranger - Expériences et projets", rapport du Centre d'étude de l'opinion publique (CBOS), février 2008. 
6. Krystyna Iglicka, "Let Us Tear Down the Curtain of Salty Water. Transatlantic Dimension of Temporary Labor Migration - Perspective of Central Europe", rapport du Center for International Relations, en coopération avec le German Marshall Fund, Varsovie, 2008, p.5, 7 et 15.

7. www.swietapolska.com/news/, 27 nov. 2007.

8. Dziennik, 27 février 2009.

9. Lena Kolarska-Bobinska, "L'image de la Pologne et des Polonais en Europe. 10 questions sur l'avenir" (en polonais), Varsovie, ISP, déc. 2003 ; “Comment améliorer l'image du Polonais en Europe occidentale?" (en polonais), www.opoka.org.pl/biblioteka/X/XU/5kolarska.html et L. Kolarska-Bobinska, La Pologne et l'Allemagne. Image réciproque et perception de l'Europe, cité par F. Ganczak, Newsweek Polska, 13 janvier 2009 ; voir aussi l'interview d'Agnieszka Lada, directrice du programme européen de l'ISP, Polska, 24 mars 2009.

10. Voir, par exemple, "Ce sont les émigrés qui vont construire une nouvelle Pologne" (en polonais), Gazeta Wyborczka, 18 juin 2006 et A. Bonnet, "Pologne : les Britanniques regrettent déjà leurs Polonais", www.regard-est.com, 28 oct. 2008.

11. Interview à www.cafebabel.com, 30 janvier 2006.

12. M. Dabrowski, “La Pologne (vue de l'UE)”, in Y. Bertoncini, T.A. Dulphy, S. Kahn, C. Manigand (dir.) Le dictionnaire critique de l'Union européenne, Paris, Armand Colin, 2008, p. 343-4.

13. Rapport de CBOS, Les étrangers qui travaillent en Pologne, mai 2008.

14. Renata Orlowska, L'influence de l'émigration des Polonais sur la conjoncture économique en Pologne, rapport, université de Gdansk, Institut du commerce avec l'étranger.

15. P. Kaczmarczyk, M. Okolski, Economic Impact of Migration on Poland and the Baltic States, Fafopaper, 2008.

16. Tygodnik Powszechny, 6 janv. 2008, p. 3-6.

17. Rapport de CBOS de février 2008.

18. Iza Chmielewska, Grzegorz Dobroczek, "Funds Transferred to Poland", intervention déjà citée lors du colloque à Varsovie, 8-9 déc. 2008.

19. Dominika Blachnicka, La dernière vague d'émigration..., op. cit.

20. T. Rozek, Polska, 5 juillet 2008 ; Rzeczpospolita, 28 nov. 2008.

21. A. Fihel, M. Okolski, “Case study...”, op. cit., p. 18.

22. PAP, Dziennik, 8 avril 2009.

23. Miroslaw Bieniecki, Justyna Frelak, Piotr Kazmierczak, Le travail des étrangers en Pologne est une solution aux problèmes du marché du travail, Institut Spraw Publicznych, Analisy i Opinie, mai 2007 ; "L'Est sur les chantiers", Ozon, 22 mars 2006.

24. Philippe Rusin, "Élargissement et flux migratoires...", art. cit.

25. A. Fihel, M. Okolski, “Case study...”, op. cit. ; Ph. Rusin, "Élargissement et flux migratoires....", op. cit., p. 29 ; GUS, La situation démographique de la Pologne, annuaire, Varsovie, 2007.

26. Tygodnik Powszechny, 11 mars 2009.

27. World Bank, "In Focus : an Update on Labor Migration from Poland", 10 oct. 2008.

28. Moniak Stelmach, "Retour ou marche arrière", Polityka, 2 août 2008, pp. 86-89.

29. Rzeczpospolita, 24 nov. 2008.

30. Rzeczpospolita, 9 janvier 2009.

31. Ali Mansoor, Bryce Quillin (Dir.), Migration and Remittances - Eastern Europe and the Former Soviet Union, The World Bank, 2006 ; voir aussi Rzeczpospolita, 24 nov. 2008.

32. Magdalena Lesinska, "Sur la politique migratoire", Biuletyn Migracyjny, janv. 2008 ; Miroslav Bieniecki, Justyna Frelak, Piotr Kazmierczak, Le travail des étrangers en Pologne est une solution pour régler les problèmes du marché du travail, Varsovie, Institut des affaires publiques, mai 2007 ; Rzeczpospolita, 31 mars 2009.

33. Voir, par ex., "The Polish Dream Turns Sour", The Independent, 4 mars 2009. 
34. Ex. B. Coles, "Exodus : Why the Poles Are Going Home", The Daily Express, 18 oct. 2008 ; M. Bolger, "These Three, and Hundreds of Thousands Like Them, Are Planning to Go Home", Building, 24 octobre 2008.

35. Cité par Building, 24 oct. 2008.

36. Dziennik, 30 janvier 2009.

37. Warsaw Voice, 19 mai 08 et Associated Press, 22 sept. 2008.

38. http://www.metiseurope.eu, 1er mars 2008 et www.swietapolska.com/news/, 26 avril 2008 ; The Times, 16 mars 2009 ; The Herald, 25 fev. 2009.

39. Warsaw Voice, 24 déc. 2008.

40. Rapport sur l'émigration de la Fédération des Polonais de Grande-Bretagne, 29 janv. 2009 ; Rzeczpospolita, 30 janvier 2009.

41. Citée par The Daily Telegraph, 14 fév. 2009 et Rzeczpospolita, 30 janvier 2009.

42. Dziennik, 30 janvier 2009.

43. Catherine Glossop \& Faiza Shaheen, Accession to Recession...", op. cit., mars 2009, p. 24 ; Financial Times, 16 mars 2009 ; Guardian Unlimited, 16 mars 2009; The Times, 16 mars 2009. Le salaire hebdomadaire moyen des Européens de l'Est serait de 290 livres contre 436 livres au niveau national, The Daily Telegraph, 14 fév. 2009.

44. Rzeczpospolita, 30 janvier 2009.

45. Dziennik, 26 et 27 mars 2009 ; Rzeczpospolita, 9 mars 2009 ; Gazeta Wyborcza, 11 février 2009.

46. Rzecpospolita, 9 mars 2009 .

\section{RÉSUMÉS}

L'adhésion de la Pologne à l'UE, le 1er mai 2004, a été suivie du départ en Grande-Bretagne et en Irlande de centaines de milliers de Polonais en quête de travail. Face au chômage et à la pauvreté en Pologne, ils sont poussés par l'espoir de meilleurs salaires. D'autant que les Polonais, ressortissants d'un nouvel État membre, ont immédiatement eu accès aux marchés du travail de ces "îles". Si les capitaux qu'ils transfèrent en Pologne servent surtout l'économie domestique, les migrants contribuent depuis à l'évolution des représentations qui déterminent le dialogue et la coopération entre la Pologne et les autres pays de l'Union européenne.

\section{AUTEURS}

\section{ANNE DE TINGUY}

Professeur des universités, chercheur au CNRS (CERI Sciences Po).

\section{CATHERINE WIHTOL DE WENDEN}

Directrice de recherche au CNRS (CERI Sciences Po). 